\title{
Induced neural stem cells: a new tool for studying neural development and neurological disorders
}

\author{
Guang-Hui Liu ${ }^{1, *}$, Fei Yi ${ }^{2, *}$, Keiichiro Suzuki², Jing Qu ${ }^{1,2}$, Juan Carlos Izpisua Belmonte ${ }^{2,3}$ \\ ${ }^{I}$ National Laboratory of Biomacromolecules, Institute of Biophysics, Chinese Academy of Sciences, Beijing 100101, China; ${ }^{2}$ Gene \\ Expression Laboratory, Salk Institute for Biological Studies, 10010 North Torrey Pines Road, La Jolla, CA 92037, USA; ${ }^{3}$ Center \\ for Regenerative Medicine in Barcelona, Dr. Aiguader 88, 08003 Barcelona, Spain
}

Cell Research (2012) 22:1087-1091. doi:10.1038/cr.2012.73; published online 1 May 2012

Recent advances in the genera-
tion of multipotent and expandable
induced neural stem cells are excit-
ing. They not only hold great prom-
ises for potential clinical applications
but may also open up a new era for
neural stem cell research in the near
future.
Cell-based therapy for treating neurodegenerative diseases is a primary goal in regenerative medicine research that attracts tremendous attention. Advances in the direct lineage conversion of somatic cells into induced neurons (iNs) in vitro provides an interesting alternative to induced pluripotent stem cell (iPSC) based disease modeling. However, the innate limitations of iNs may restrict their applications in the clinic. Very recent publications describe the direct conversion of mouse somatic cells into induced neural stem cells (iNSCs) [1-6]. Together with other reports $[7,8]$, they constitute the basis for a rising concept that priming and retaining reprogrammed cells into an expandable neural progenitor stage may render them to be more amenable and with great

\footnotetext{
*These two authors contributed equally to this work.

Correspondence: Guang-Hui Liua, Juan Carlos Izpisua Belmonte ${ }^{\mathrm{b}}$

aE-mail: ghliu@ibp.ac.cn

bE-mail: belmonte@salk.edu, izpisua@cmrb.eu
}

potential for both disease modeling and possible therapeutic treatment for neurological diseases.

Whereas the general concept is similar among these studies, they differ in methodology (Table 1). In 2011, Kim et al. [1] first reported direct reprogramming of mouse fibroblasts to neural progenitors. Using a doxycycline (dox)inducible secondary mouse embryonic fibroblast (MEF) system, the authors found that transient induction of four Yamanaka factors reprogrammed fibroblasts into iNSCs under neural inductive conditions. These iNSCs homogenously expressed the neural stem cell (NSC) marker Pax6 and neural rosette marker PLZF. Moreover, they efficiently differentiated into mature neurons and astrocytes in vitro. However, the iNSCs generated by Kim et al. exhibited limited proliferation potential, which likely resulted from an unstable intermediate state induced by the four "Yamanaka factors". Since then, multiple studies have been carried out aiming to improve the methodology for expandable iNSCs. Based on a screening that was successful in generating of iNs, Sheng et al. [4] conducted a reprogramming study using a selected pool of Sox $2 / \mathrm{c}-\mathrm{Myc} / \mathrm{Klf} 4$ together with another 6 neural inductive factors. The authors demonstrated that 9 factors sufficiently converted mesoderm-origin mouse sertoli cells into iNSCs colonies. These iNSCs were multipotent in vitro, and could be efficiently expanded as either a monolayer or neural spheres. Interestingly, none of the 9 factors, except for Sox2, was dispensable for the conversion. Remarkably, when transplanted into the mouse brain, the iNSCs successfully grafted and committed to neural lineages in vivo. Importantly, on the issue of iNSCs safety, no signs of tumorigenesis were observed post transplantation in vivo. Later, the same strategy was successfully applied to mouse tail tip fibroblasts and generated iNSCs with neurogenesis potency both in vitro and in vivo, which further confirmed the feasibility of this method [6]. Meanwhile, Wernig and colleagues also reported a similar screening of 11 factors using a Sox2-EGFP reporter MEF system, and found that as few as two factors, FoxG1 and Sox2, were able to generate selfrenewing, bipotent iNSCs [3]. When Brn2 was added to the combination, tripotent iNSCs, which gave rise to functional neurons, astrocytes as well as oligodendrocytes, were obtained [3]. Most recently, appearing side by side, Han et al. [2] and Thier et al. [5] further investigated the methodology of direct conversion of mouse fibroblasts into expandable, multipotent iNSCs. Han et al. [2] reported that the combination of Brn $4+$ Sox $2+\mathrm{Klf} 4+\mathrm{c}-\mathrm{Myc}$, with the 


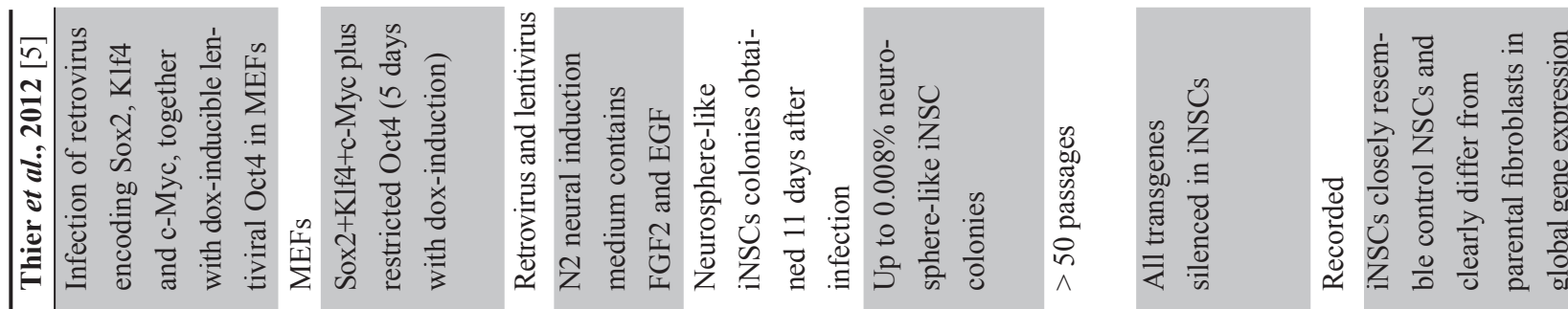

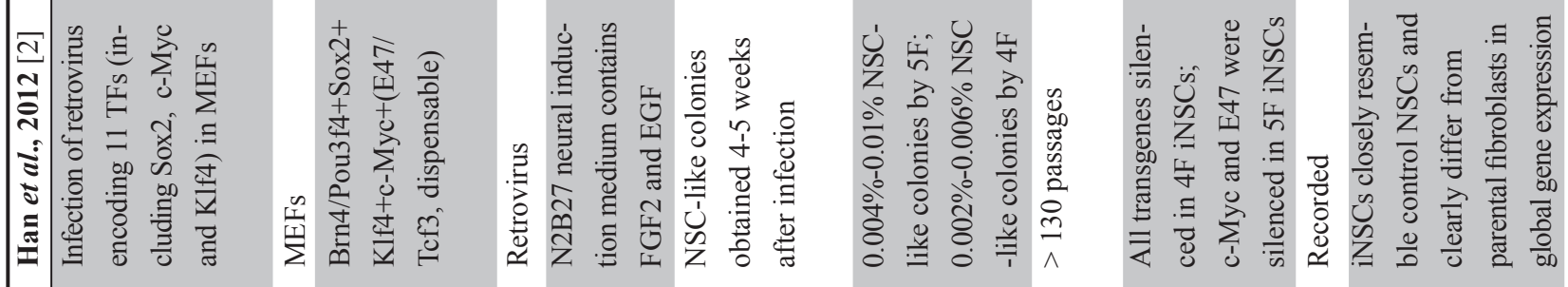

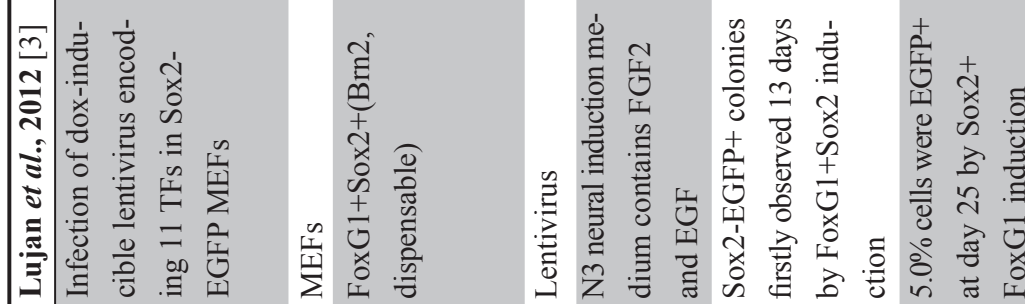

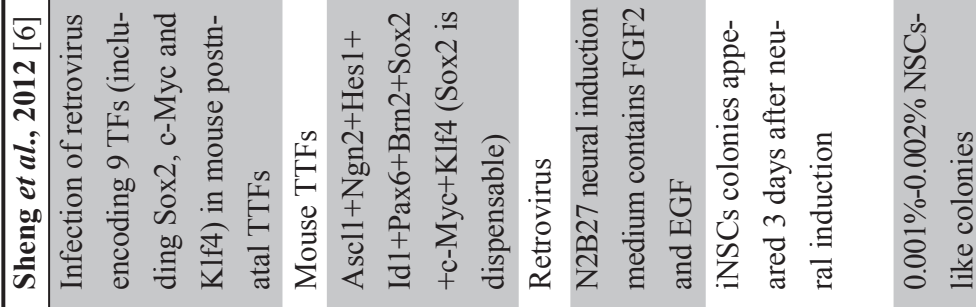

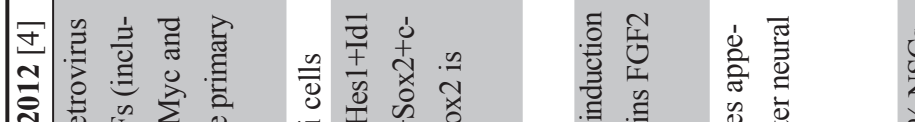

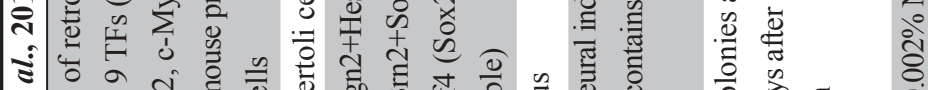

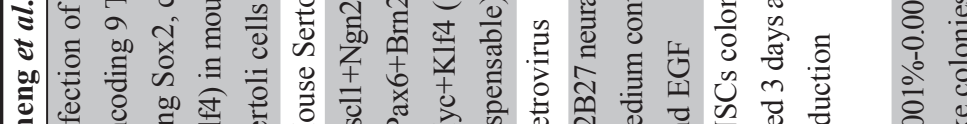

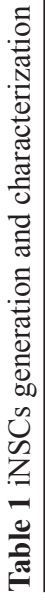
ॠ

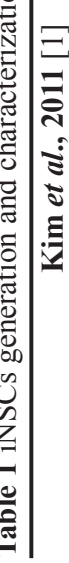
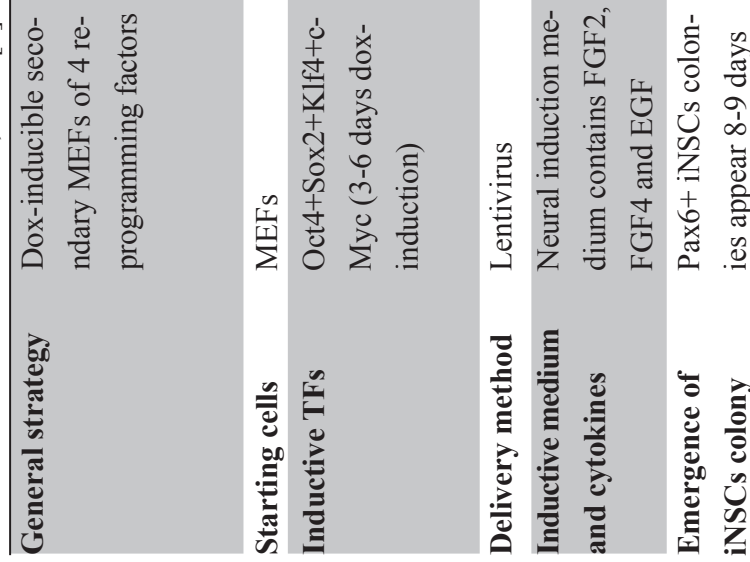

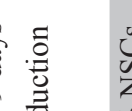

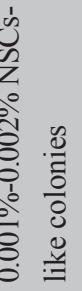

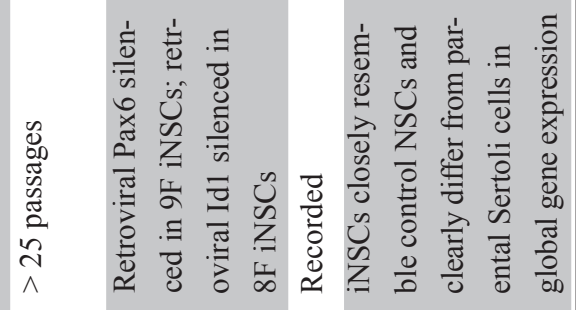

胥

$\approx$

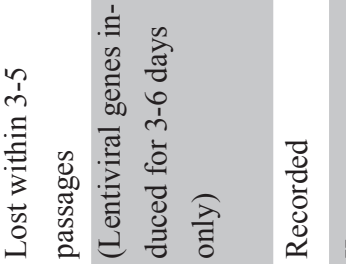

$\begin{array}{ll}0 \\ 0 \\ 1 & 0 \\ 0 & 0 \\ 0 & 0 \\ 0 & 0\end{array}$

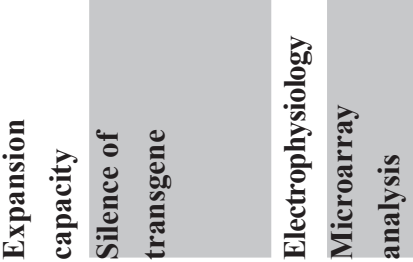




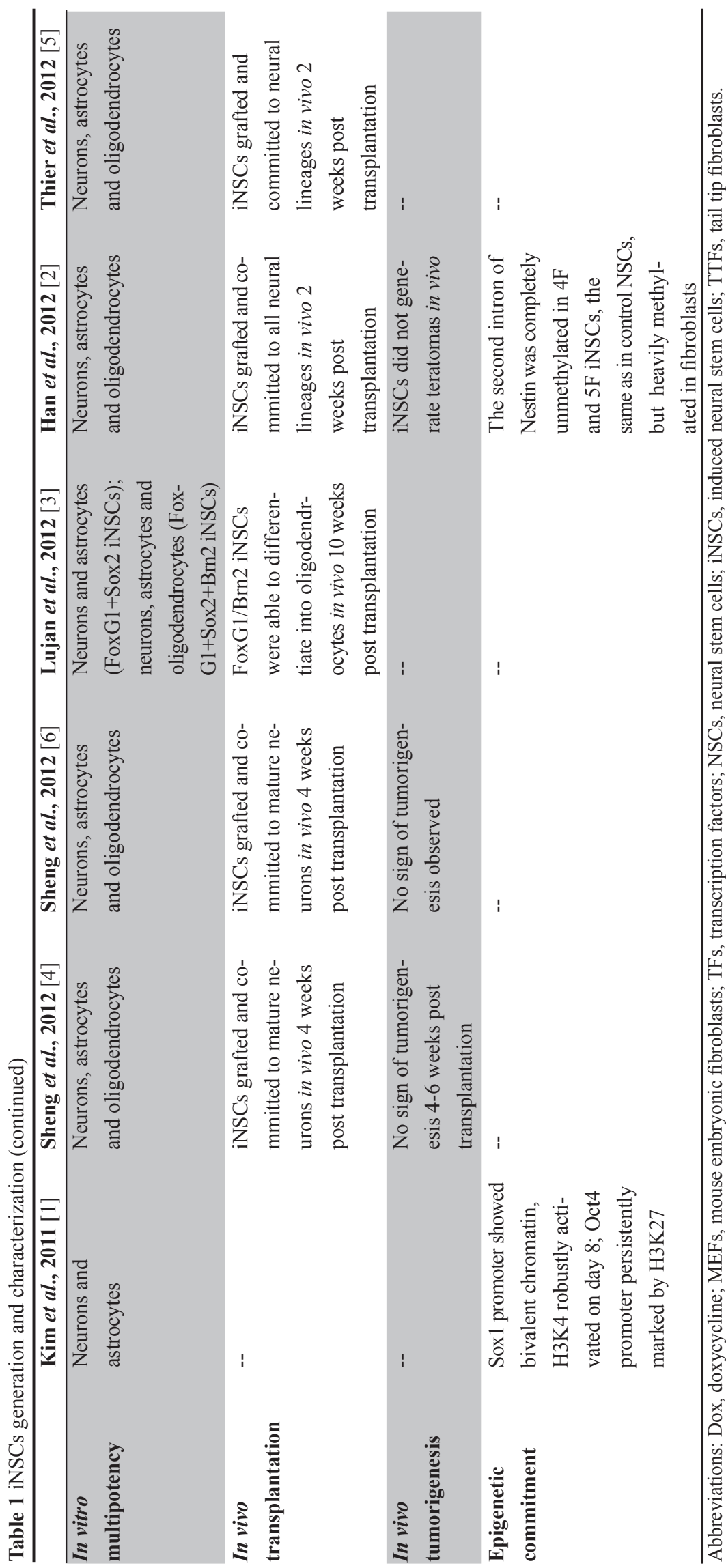

optional inclusion of E47, was sufficient to generate multipotent iNSCs, which could be maintained for more than 130 passages. Similar to Kim et al.'s finding, Thier et al. [5] reported that, by slightly manipulating expression of four Yamanaka factors (constitutively inducing Sox2, Klf4 and c-Myc while transiently priming with Oct4), neurosphere-like colonies could be obtained and expanded to multipotent iNSCs. Established iNSCs from both studies exhibited similar patterns of global gene expression when compared to normal NSCs and were able to undergo neural differentiation in vivo $[2,5]$.

There are several obvious advantages of obtaining expandable iNSCs instead of fully committed iNs or iPSC-derived NSCs. First, neurons directly converted from fibroblasts are not suitable to be used for cell transplantation due to their high heterogeneity and terminal differentiation. The iNSCs studies indicate the feasibility of generating a pure cell line potentially transplantable. Taking Han et al.'s study as an example, the iNSCs established are a homogenous cell population derived from a single colony [2]. Having multiple iNSCs lines would allow the opportunity to choose the best and most appropriate clones for both in vitro and in vivo studies. A pure population of iNSCs can also be more readily used for genome-wide analysis, such as DNA methylation, histone modification, microarrays, proteomic analysis, pull-down experiments, etc., which, similar to iPSCs, will provide an ideal platform for basic research. Moreover, iPSCs studies indicate that long-term in vitro culture may cause accumulated chromosomal abnormalities [7]. Generation of iNSCs is not only faster but also bypasses the "induced pluripotency" stage, which may help to reduce risks of tumorigenesis. Furthermore, direct generation of homogenous iNSCs avoids the potential contamination of carcinogenic iPSCs.

Second, iNSCs have been proven to be capable of self-renewal. Most iNSCs 
established by reported methods are expandable, which provides the possibility of generating a large amount of cells at the neural progenitor stage for subsequent differentiation or manipulation. In contrast, iNs directly converted from fibroblasts are terminally committed neurons that are not expandable [9]. On the other hand, the in vitro cultured patient iNSCs can be used to study the specific phenotypes of disease-affected NSCs, which are impossible to obtain from patients directly. In addition, the self-renewal activity of iNSCs enables the use of non-integrative reprogramming carriers. To date, almost all of the reported iNs are generated with lentiviral vectors, which may cause genome instability. Although viral vectors are also used for gene delivery in current iNSCs studies, it has been found that the retrovirus-mediated transgenes were frequently silenced in established iNSCs, resembling iPSCs (Table 1). The fact that iNSCs are self-renewable also provides the possibility of using episomal vectors [10], which would be diluted and ultimately eliminated as cells continuously divide during reprogramming.

Third, iNSCs have the potential to eliminate aging-related hallmarks from the original somatic cells. It has been found that reprogramming of fibroblasts into iPSCs resets cell's clock, thus generating "rejuvenated" cells endowed with several advantages for both basic research and therapeutic applications [11]. In contrast, direct conversion of fibroblasts into neurons is unable to remove the aging marks of original cells. As post-mitotic cells, neurons do not divide, and therefore they cannot dilute the toxic proteins through cell cycle progression. In this regard, the iNs directly converted from aged or diseased fibroblasts would, in principle, retain the toxic or pathogenic metabolites. For example, fibroblasts of Alzheimer's disease patient bear increased $A \beta$ protein, which would likely be retained or even amplified in iNs directly converted from them [12]. Since iNSCs regain the capability to quickly proliferate, the aging hallmarks would be easily removed with increased passaging, resulting in a reset "healthy" status more suitable for transplantation. It will be interesting to further examine various aging-related parameters such as telomere length, nuclear architecture, etc. in the generated iNSCs.

Last, most gene editing approaches used for the human genome require clonal expansion of targeted cells. Targeted gene editing technologies have recently been successfully applied in iPSCs due to their clonal expansion capability [13-16]. However, current methodologies of direct cell lineage conversion toward terminally committed cells would not be able to provide desired cell types for gene targeting. Again, due to their self-renewal capacity, iNSCs would potentially serve well as cell carriers for gene correction to eliminate and correct pathological mutations in patient cells.

In summary, though generation of iNSCs provides additional advantages over iNs towards both basic research and clinical applications, there are several important questions that remain to be answered, including 1) Is the epigenome of iNSCs stably reset to a NSC-like status? 2) Are the retroviral vectors used completely silenced in the established iNSCs and their neural derivatives? 3) Is the iNSC reprogramming process free of genomic alterations? In fact, it has been shown that NSCs derived from human embryonic stem cells have abnormal karyotypes with long-term culture in vitro [17]. In this regard, safety issues would need to be carefully evaluated in iNSCs and their derivatives before cell therapy applications can be considered. At this point, perhaps the most immediate challenge in this field would be the generation of expandable, multipotent human iNSCs. Recently, successful conversion from human astrocytes to
iNSCs has been reported [18]. Though this method is less likely to be applicable in a clinical setting, due to the obvious barriers of isolating patient astrocytes, an improved strategy using more readily available human somatic resources would be desirable in the near future.

\section{Acknowledgments}

GHL was supported by 100 Talents Program of Chinese Academy of Sciences, a CIRM grant, and a Glenn aging foundation grant. KS was partially supported by the Uehara Memorial Foundation research fellowship. JQ was partially supported by an AFAR/ Ellison Medical Foundation postdoctoral fellowship. Work in the laboratory of JCIB was supported by MICINN, Sanofi-Aventis, The Helmsley Charitable Trust, The G Harold and Leila Y Mathers Charitable Foundation and The Ellison Medical Foundation.

\section{References}

1 Kim J, Efe JA, Zhu S, et al. Direct reprogramming of mouse fibroblasts to neural progenitors. Proc Natl Acad Sci USA 2011; 108:7838-7843.

2 Han DW, Tapia N, Hermann A, et al. Direct reprogramming of fibroblasts into neural stem cells by defined factors. Cell Stem Cell 2012; 10:465-472.

3 Lujan E, Chanda S, Ahlenius H, Sudhof TC, Wernig M. Direct conversion of mouse fibroblasts to self-renewing, tripotent neural precursor cells. Proc Natl Acad Sci USA 2012; 109:25272532.

4 Sheng C, Zheng Q, Wu J, et al. Direct reprogramming of Sertoli cells into multipotent neural stem cells by defined factors. Cell Res 2012; 22:208218.

5 Thier M, Wörsdörfer P, Lakes YB, et al. Direct conversion of fibroblasts into stably expandable neural stem cells. Cell Stem Cell 2012; 10:473-479.

6 Sheng C, Zheng Q, Wu J, et al. Generation of dopaminergic neurons directly from mouse fibroblasts and fibroblastderived neural progenitors. Cell Res 2012; 22:769-772.

7 Panopoulos AD, Ruiz S, Izpisua Belmonte JC. iPSCs: induced back to controversy. Cell Stem Cell 2011; 8:347348 . 
8 Zhang W, Duan S, Li Y, et al. Converted neural cells: induced to a cure? Protein Cell 2012; 3:91-97.

9 Yang N, Ng YH, Pang ZP, Sudhof TC, Wernig M. Induced neuronal cells: how to make and define a neuron. Cell Stem Cell 2011; 9:517-525.

10 Okita K, Matsumura Y, Sato Y, et al. A more efficient method to generate integration-free human iPS cells. Nat Methods 2011; 8:409-412.

11 Liu GH, Barkho BZ, Ruiz S, et al. Recapitulation of premature ageing with iPSCs from Hutchinson-Gilford progeria syndrome. Nature 2011; 472:221225.

12 Qiang L, Fujita R, Yamashita T, et al.
Directed conversion of Alzheimer's disease patient skin fibroblasts into functional neurons. Cell 2011; 146:359371.

13 Pan H, Zhang W, Liu GH. Find and replace: editing human genome in pluripotent stem cells. Protein Cell 2011; 2:950-956.

14 Liu GH, Sancho-Martinez I, Izpisua Belmonte JC. Cut and paste: restoring cellular function by gene correction. Cell Res 2012; 22:283-284.

15 Soldner F, Laganière $\mathrm{J}$, Cheng AW, et al. Generation of isogenic pluripotent stem cells differing exclusively at two early onset Parkinson point mutations. Cell 2011; 146:318-331.
16 Liu GH, Suzuki K, Qu J, et al. Targeted gene correction of laminopathyassociated LMNA mutations in patientspecific iPSCs. Cell Stem Cell 2011; 8:688-694.

17 Varela C, Denis JA, Polentes J, et al. Recurrent genomic instability of chromosome $1 \mathrm{q}$ in neural derivatives of human embryonic stem cells. J Clin Invest 2012; 122:569-574.

18 Corti S, Nizzardo M, Simone C, et al. Direct reprogramming of human astrocytes into neural stem cells and neurons. Exp Cell Res 2012; 318:15281541. 